\title{
Erratum to: A geometric Ginzburg-Landau problem
}

\section{Roger Moser}

Published online: 27 November 2013

C Springer-Verlag Berlin Heidelberg 2013

\section{Erratum to: Math. Z. (2013) 273:771-792}

DOI 10.1007/s00209-012-1029-5

The value of the constant $c$ in Theorem 1.1 of the paper [1] is incorrect. The correct value is

$$
c=4 \pi \sqrt{\frac{2}{3}} .
$$

The mistake comes from a confusion of the exponent $-\frac{2}{3}$ and $-\frac{3}{2}$ in the proof, from p. 776 onwards. The methods used in the proof are correct, but several of the calculations need to be modified. In Sect. 2.2, the definition of the function $g$ should be

$$
g(s)=\int_{0}^{s} \frac{d r}{\sqrt{r^{-2 / 3}-1}},
$$

or equivalently,

$$
g(s)=2-\left(s^{2 / 3}+2\right) \sqrt{1-s^{2 / 3}} .
$$

This will yield $\ell=\frac{4}{3}$ instead of the value given. In Sect. 2.4, with the same methods as in the paper, and using

$$
\int_{0}^{1} \frac{d s}{s^{2 / 3} \sqrt{s^{-2 / 3}-1}}=\left[-3 \sqrt{1-s^{2 / 3}}\right]_{0}^{1}=3,
$$

The online version of the original article can be found under doi:10.1007/s00209-012-1029-5.

R. Moser $(\bowtie)$

Department of Mathematical Sciences,

University of Bath, Bath BA2 7AY, UK

e-mail: r.moser@bath.ac.uk 
we obtain

$$
c=\frac{F(v)}{\sqrt{2 \ell}}=4 \pi \sqrt{\frac{2}{3}} .
$$

In Sect. 2.3, the fraction $\frac{7}{4}$ has to be replaced by $\frac{4}{3}$ throughout. The methods still work and prove statement (4) of the paper for $\sigma<\frac{1}{2}$, which is sufficient for the proof of Theorem 1.1 with the correct constant.

\section{Reference}

1. Moser, R.: A geometric Ginzburg-Landau problem. Math. Z. 273, 771-792 (2013) 